Meta

Journal des tradlucteurs

Translators' Journal

\title{
Déclaration concernant la formation des interprètes et des traducteurs
}

\section{Frank G. Königs}

Volume 39, numéro 3, septembre 1994

URI : https://id.erudit.org/iderudit/003338ar

DOI : https://doi.org/10.7202/003338ar

Aller au sommaire du numéro

Éditeur(s)

Les Presses de l'Université de Montréal

ISSN

0026-0452 (imprimé)

1492-1421 (numérique)

Découvrir la revue

Citer cette note

Königs, F. G. (1994). Déclaration concernant la formation des interprètes et des traducteurs. Meta, 39(3), 533-533. https://doi.org/10.7202/003338ar d'utilisation que vous pouvez consulter en ligne.

https://apropos.erudit.org/fr/usagers/politique-dutilisation/ 


\section{DÉCLARATION CONCERNANT LA FORMATION DES INTERPRĖTES ET DES TRADUCTEURS}

À la suite d'une invitation de l'Institut Goethe et en coopération avec le Sprachen-und DolmetscherInstitut de Munich eut lieu, du 17 au 21 décembre 1993, un séminaire de travail concernant la formation des interprètes et des traducteurs. Les participants à ce séminaire, des enseignants chargés de la formation des interprètes et des traducteurs venant de 18 pays, ont convenu de la déclaration suivante :

- Les exigences professionnelles que rencontrent interprètes et traducteurs requièrent une professionnalisation et un fondement scientifique dans le domaine de la traduction et de l'interprétariat ainsi que dans la formation y préparant. Cette formation doit être attachée aux institutions reconnues qualifiées pour ce faire et y être dispensée par des formateurs spécialisés en la matière, y compris du point de vue didactique. Dans la mesure où de telles institutions n'existent pas encore, il faut les creer. Les institutions et organisations existantes sont appelées à y apporter leur appui.

- La formation dans le domaine de l'interprétariat et de la traduction doit fondamentalement se distinguer de la formation du professeur de langue ainsi que de celle ayant pour objectif I'enseignement d'une langue étrangère. L'acquisition de compétences linguistiques dans le cadre de l'apprentissage d'une langue étrangère est, certes, une condition nécessaire à la réalisation des objectifs de formation dans le domaine mentionné plus haut et doit, en conséquence, être réalisée dans un cadre institutionnel, mais elle doit nettement se différencier de l'acquisition d'une compétence absolument spécifique relative à l'interprétariat et à la traduction. Les différents cursus de formation peuvent se combiner entre eux tout en conservant leurs objectifs de formation spécifique dans la mesure où se trouvent aménagées des possibilités de transition adéquates. Les programmes d'enseignement doivent être organisés de façon à permettre l'obtention de certifications d'études diversifiées à différents niveaux et à laisser la voie ouverte à une combinaison de modules de formation dans le domaine de la traduction et de l'interprétariat.

Les participants au séminaire de travail «Formation des interprètes»

(Texte communiqué par FRANK G. KÖNIGS) Ruhr-Universität Bochum, Allemagne 